\title{
Refractory Cardiogenic Shock Induced by Influenza
}

\section{Type B}

\author{
AZHARI Alaa ${ }^{1}$ *MD, D’OSTREVY Nicolas ${ }^{1}$, AZARNOUSH Kasra MD PHD ${ }^{1}$, Geoffroy Etienne ${ }^{1}$, \\ MEDARD Anne MD ${ }^{1}$, ULMANN Lucie ${ }^{1}$, CAMILLERI Lionel MD PHD ${ }^{1}$
}

\author{
${ }^{1}$ Department of Cardiovascular surgery, G. Montpied, Clermont-Ferrand University Hospital, France
}

\begin{abstract}
Introduction: Good health education among patients and health professionals with updated preventive vaccination for myocarditis can improve the survival outcome. In addition to this, Knowing the indications of ECMO in case of resuscitation with a good installation in an appropriate timing will prevent multiple organ failure and lead to rapid recovery with short duration use of Extracorporeal Life Support, this case report is rare and there is just a few cases reported in 2013 and we do an improvement in our management. Case presentation: A 49 year-old-woman without known heart disease was referred to our university hospital emergency department after 3 days of Influenza symptoms. At her arrival, rapid-onset cardiogenic shock with normal left ventricular size,diffuse left ventricular hypokinesia, and an ejection fraction of 5\% are confirmed by Trans-thoracic echocardiography. Coronary angiography was normal. Echocardiography shows no valves abnormality. However, the patient gets in cardiorespiratory arrest. So, an external cardiac massage was performed then an Extracorporeal Life Support was required. A nasal swap at the admission was positive for influenza type $B$ virus. All other respiratory, blood and urine cultures were negative. On day 8 , a repeated Trans-thoracic echocardiography demonstrated significant recovery of left ventricular function with ejection fraction of 20\%. Then, full recovery on day 12 with ejection fraction of 50\%. Conclusions: Influenza type $B$ infection can cause a fulminant cardiomyopathy without preexisting cardiac disease which can leads to refractory cardiogenic shock. ECMO is a suitable choice for fulminant myocarditis.
\end{abstract}

\section{List of abbreviations:}

Fulminant Myocarditis (FM), ElectroCardioGram (ECG), Extracorporeal Life Support (ECLS),

Emergency Room (ER), Blood Pressure (BP), IntraVenous (IV), Intensive Care Unit (ICU),Arterial Blood Gas (ABG), Trans-Esophageal Echocardiography (TEE), Left Ventricular (LV),Left Ventricular Ejection Fraction (LVEF), Cardiac Index (CI), White Blood Cell (WBC),Sodium (Na), Potassium (K), Chloride (Cl), C-Reactive Protein (CRP), Creatinine (Cr),Aspartate Aminotransferase (AST), Alanine Aminotransferase (ALT), Trans-ThoracicEchocardiography (TTE), Capillary Wedge Pressure (PCW), Transapical Left Ventricle Vent(TLVV), Broncho alveolar lavage (BAL), Ejection Fraction (EF), World Health Organization(WHO), Cardio-Pulmonary Resuscitation (CPR), Ventricular Assisted Device (VAD), Bi-Ventricular Assisted Device (BiVAD).

Keywords: Influenza B, Viral Myocarditis, Fulminant Myocarditis, Cardiogenic Shock, Extracorporeal Membrane Oxygenation ECMO, Biventricular assisted device BiVAD.

\section{Introduction}

Influenza, is an infectious disease caused by the influenza virus. It's a common febrile illness during winter. Myocarditis mostly caused by Coxackie B virus, influenza viruses, and echoviruses and it's a self-restricting ending with full recovery, but in the other hand can leads to heart failure [1]. The incidence of myocarditis differs between influenza A (1.3\%), B (0.7\%) and C (3.6\%) virus infections [2]. Fulminant myocarditis (FM) usually following a flu-like illness characterized by sudden onset of severe congestive heart failure or cardiogenic shock [1]. Diagnosis should exclude coronary arteries and acute valvular diseases [2]. The clinical manifestations of viral myocarditis are varying greatly from asymptomatic electrocardiogram (ECG) to severe heart failure [3].

We report a notable case of profound cardiogenic shock of 49 year old female due to influenza virus type B leaded to FMwith no known pre-existing heart disease. She was successfully managed with Extracorporeal Life Support (ECL).

\section{Case Report}

A 49 year-old-woman without known heart disease, presented in the emergency room (ER) with profound cardiogenic shock. The past 3 days prior to admission she had fever, shivering, chills, diffuse myalgia, nausea and vomiting, diagnosed by her doctor as an influenza. The following days, she had a severe chest and epigastric pain associated with many vomiting episodes. A very low blood pressure (BP) $(60 / 40 \mathrm{mmHg})$ even after a $2 \mathrm{~L}$ of intravenous (IV) fluid injection for hemodynamic resuscitation justified her admission in intensive care unit (ICU), associated to an impaired level of consciousness, fever, mottled skin and sinus tachycardia without a radial pulse.The ECG revealed an accelerated sinus rhythm with a rate of 140 beats/min, with diffuse low voltage. Arterial blood gas (ABG) showed a severe metabolic acidosis with $7.14 \mathrm{PH}$, Lactate $7.8, \mathrm{HCO}_{3}$ 8 and anuria. Examination revealed: A bilateral lung crepitation, and a benign abdomen. On emergent TEE left ventricular (LV) function was severely depressed witha normal dimension and global hypokinesia, an estimated left ventricular ejection fraction (LVEF) of 5\%with a cardiac index (CI) of $1.7 \mathrm{~L} / \mathrm{min} / \mathrm{m} 2$, justifying an emergent coronarography which showed normal coronary arteries (Figure 2) and severe LV impairment. 


\section{International Journal of Science and Research (IJSR) \\ ISSN (Online): 2319-7064}

Index Copernicus Value (2013): 6.14 | Impact Factor (2015): 6.391

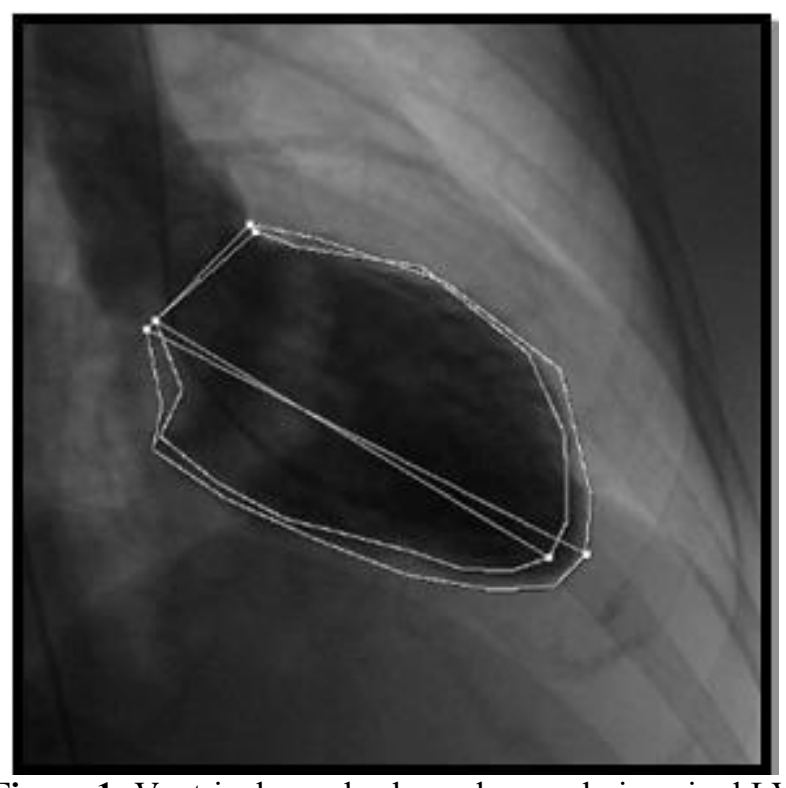

Figure 1: Ventriculographyshowed severely impaired LV function with normal dimension, global hypokinesia and LVEF of $5 \%$

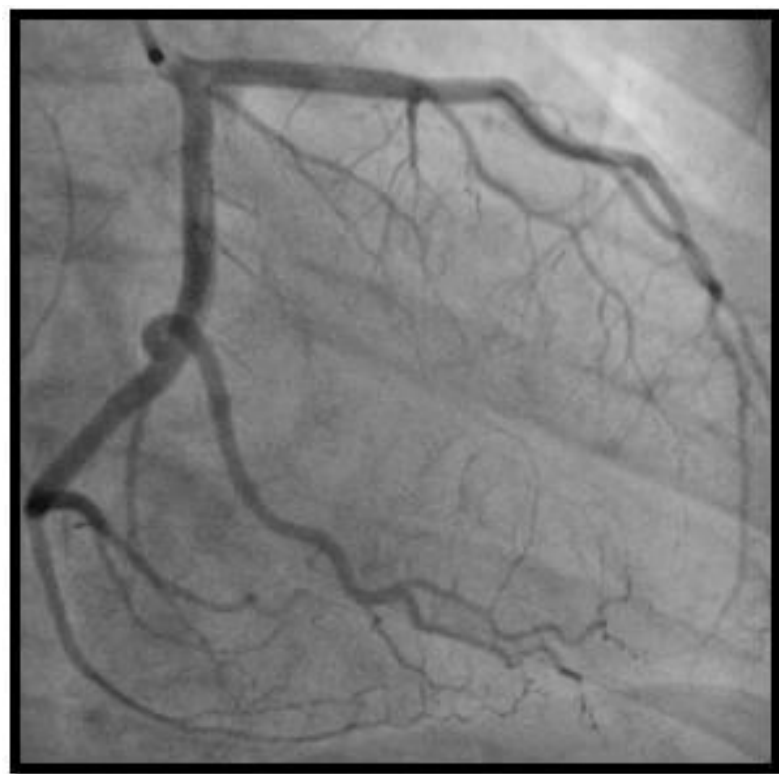

Figure 2: Coronarography shows anormal coronary arteries (left-dominant)

Soon, the patient went to a cardiorespiratory arrest. So, she was intubated, sedated during external cardiac massage with an absence of no-flow (low-flow 10 minutes). Three mg of Adrenaline was given, then an emergent peripheral venoarterial ECLS was placed via left common femoral vein and artery with leg reinjection (flow $=4 \mathrm{~L} / \mathrm{min}$ ). Initial labs revealed : white blood cell count (WBC) 7.34 Giga/L with $79.1 \%$ neutrophils, hemoglobin $11.2 \mathrm{~g} / \mathrm{dl}$, hematocrit $34.9 \%$, platelets 160 , lactate $7.8 \mathrm{mmol} / \mathrm{L}$, sodium $(\mathrm{Na}) 150 \mathrm{mmol} / \mathrm{L}$, potassium $(\mathrm{K}) 4.0 \mathrm{mmol} / \mathrm{L}$, chloride $(\mathrm{Cl})$ 124, C-reactive protein (CRP) $2.5 \mathrm{mg} / \mathrm{L}$, creatinine $(\mathrm{Cr}) 1.443 \mathrm{mg} / \mathrm{dl}$, blood glucose $319.86 \mathrm{mg} / \mathrm{dl}$, aspartate aminotransferase (AST) 32 IU/L, alanine aminotransferase (ALT) 30 and serum troponin I $3.25 \mathrm{ng} / \mathrm{ml}$. Influenza virus type B was detected in the mucus from her nasal cavity by a nasal swap that have been done at the time of admission. All other respiratory, blood and urine cultures were negative.Day one post ECLS, a repeat Trans-thoracic echocardiography (TTE) demonstrating a LVEF of 5\%, normal ventricles dimensions, without any valvulopathy, a permanently closed aortic valve, and global hypokinesiaof LV with minor regional variation and signs of stagnation in LV. In addition to TTE result, there is a radiological finding of pulmonary edema with an elevation of pulmonary capillary wedge pressure (PCW), controlled by Swan-Ganz catheterization. So, regarding to our cardiac surgery department recommendations, these findings lead us to convert peripheral ECLS to a central ECLS withtrans-apical left ventricle vent (TLVV). The ECLS was then effective between an accurately replaced venous cannula in right atrium from the right femoral vein and an arterial cannula in the right subclavian arterywith aTLVV by performing a short left thoracotomy. On day 2 of hospitalization, another repeat TTE showed persistent severe LV systolic dysfunction with EF of 5\% with a good functioning TLVV. Immunohistochemical stain for parvovirus B19, HHV6, HSV and PCR for enteroviruses, EBV and CMV were all negative. Blood, Broncho alveolar lavage (BAL) and urine cultures were negative for bacterial growth. On day 8 , a repeat echocardiography demonstrated a significant recovery of LV function with an estimated EF of $20 \%$, with a non-compressive circumferential pericardial effusion less than $5 \mathrm{~mm}$. The same day, a Staphylococcus Aureus and Pseudomonas Aeruginosa were detected in tracheal aspiration. So Colimycine, amiklin and aztreonam are given for 2 weeks. On day 16, we find an excellent recovery of LV function with a low ECLS (flow $=1.5$ $\mathrm{L} / \mathrm{min}$ ). On day 17 the ECLS ablation was successfully done. The day after, TTE revealed LVEF of $50 \%$ with cardiac output of 51/min. Totalstay was 27 days in the ICU then 12 days in the cardiac surgery ward. Finally, she discharged home with a full cardiac recovery. (Figure 3 )illustratedthe variation of the main biological results and the EF during the hospitalization.
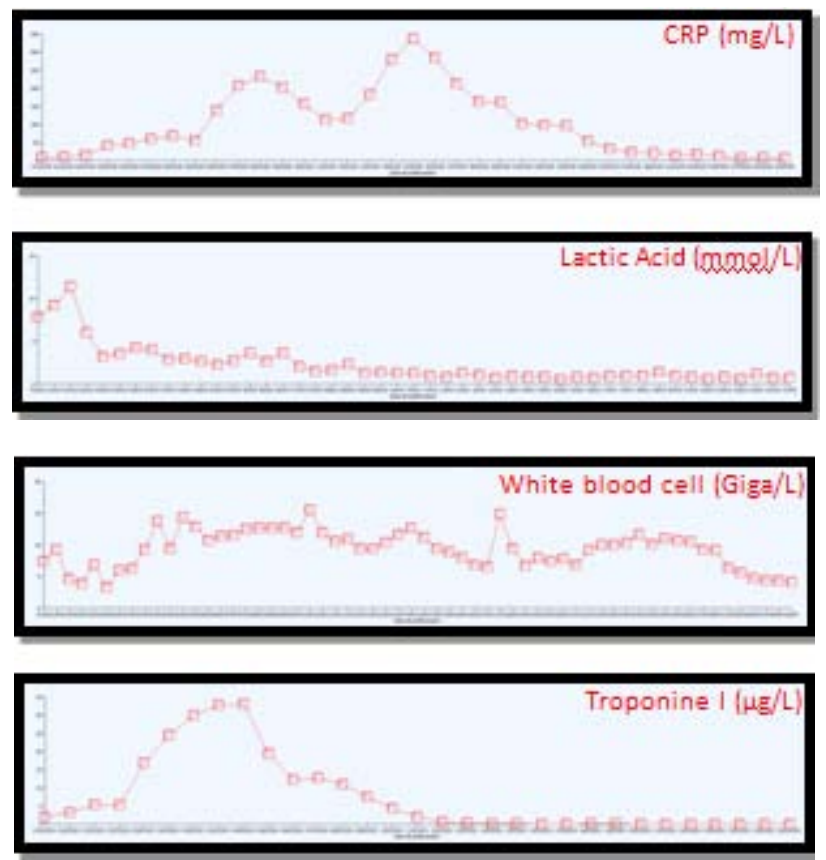

Volume 5 Issue 7, July 2016 www.ijsr.net

Licensed Under Creative Commons Attribution CC BY 


\section{International Journal of Science and Research (IJSR) \\ ISSN (Online): 2319-7064}

Index Copernicus Value (2013): 6.14 | Impact Factor (2015): 6.391



Figure 3: The variation of biological results and EF (CRP, Troponine I, Lactic acid, WBC and EF)

\section{Discussion}

The Influenza is a contagious disease caused by influenza viruses. It can cause mild to severe illness and sometimes can lead to death. So, getting the seasonal vaccination is the single best way to prevent this disease and its epidemic spreading. The Complications of influenza may include pneumonia, sinus infections, and worsening of chronic disease such as pulmonary failure, asthma or diabetes [4].

Our patient has influenza type B/Yamagata virus that had been diagnosed by a nasal swab at the day of admission. In line with the new recommendations issued by The World Health Organization (WHO) 2015/2016, they added a new strain of influenza $B$ to the trivalent vaccine[5].WHO recommends early vaccination, several weeks before seasonal influenza outbreaks, for those aged over than 65 and health Professionals [4].

FM can be differentiated from acute myocarditis which the clinical presentations are different; FM has recent influenza symptoms with a faster progression to refractory cardiogenic shock in comparison to acute myocarditis which is over several weeks. TTE is currently recommended in the initial diagnostic evaluation of all patients with suspected myocarditis.Major diffuse hypokinesiacame in both types but the typical echocardiographic finding in FMis a dilated ventricle cavity with a hypertrophy walls but in acute myocarditis ventricle cavities are normal. In long term recovery of LV function in FM within days or weeks but in acute myocarditis lead to chronic heart failure [6].

In our patient, after the cardiopulmonary arrest we start a Cardiopulmonary resuscitation (CPR), then a rapid response by our surgical team for application of a peripheral venoarterial ECLS via left common femoral vein and artery with elective limb reperfusion is successfully done. The day after, an ETT revealed an absence of ventricular ejection with ascending aortic sub valvular blood stagnation. So, conversion decision was decided by the medical-surgical team from a switch of the peripheral ECLS to central ECLS (right subclavian artery-right atrium) with TLVV by left mini thoracotomy with a direct canulation to the LV. Early LV decompression by TLVV can prevent the high LV pressure which can cause sub endocardial ischemia and impairs LV recovery [7].
Refractory cardiogenic chock secondary to FM is associated with a high mortality rate as high as $75 \%$ [8,9]. A mechanical circulatory support such as a percutaneous ECMO with a cardiopulmonary bypass or ventricular assisted device (VAD) can revert this fulminant course. So, early recognition with an immediate rescue can increase the overall survival rate range of $50-70 \%$ by cardiac recovery or even cardiac transplantation [10]. The studies showed that percutaneous ECMO has a higher survival rate than VAD [11]. This due to many advantages of percutaneous ECMO on VAD. First, quicker and easier application preventing the multiple organ failure secondary to haemodynamic deterioration. Secondly, easier to wean off than VAD, and ECMO can be converted to VAD. Third, the support duration to recovery was shorter in ECMO than in VAD [1213]. Fourth, daily troponin level was found as a good indicator for myocardial recovery in weaning of ECMO but it cannot be applied in VAD patient [14]. On the other hand, Grinda et al reported 5 consecutive FM patients with intractable cardiogenic shock by using mechanical paracorporel (BiVAD) with a successfully bridged to recovery. They used BiVAD instead of ECMO because of a diffuse biventricular disease in FM,BiVAD will give an efficientunloading of $\mathrm{LV}$ witch is important for rapid recovery [15].

In the future, good health education among patients and health professionals with updated preventive vaccination for myocarditis can improve the survival outcome in similar cases. In addition to this, Knowing the indications of ECMO in case of resucitataion with a good installation in an appropriate timing will prevent multiple organ failure and lead to rapid recovery with short duration use of ECMO.

\section{Consent:}

Written informed consent was obtained from the patient for publication of this case report. A copy of the written consent is available for review by the Editor-in-Chief of this journal.

\section{Competing Interests}

The authors declare that they have no competing interests.

\section{Author Profile}

AZHARI Alaa MD: conceived the study and performed data acquisition, data interpretation and wrote the manuscript.

D'OSTREVY Nicolas: patient management and data acquisition.

AZARNOUSH Kasra MD PHD: patient management and data acquisition.

GEOFFROY Etienne: patient management and data acquisition.

MEDARD Anne MD: patient management and data acquisition.

ULMANN Lucie: patient management and data acquisition.

CAMILLERI Lionel MD PHD: Head of cardiovascular surgery Department, study conception, final approval. 


\section{References}

[1] A. Combes *, C.-E. Luyt, J.-L. Trouillet. Acute myocarditis, puplished by Elsevier SAS 14 (2005) 248254

[2] Jun Muneuchi*, Yoshiaki Kanaya, Tomoko Takimoto, Takayuki Hoshina, Koichi Kusuhara and Toshiro Hara (June 2009). Myocarditis mimicking acute coronary syndrome following influenza B virus infection: a case report. Cases Journal 2009, 2:6809 doi: 10.4076/17571626-2-6809.

[3] Sano T1. Influenza myocarditis and pericarditis 2006 Oct;64(10):1916-20.

[4] Key Facts about Influenza (Flu) \& Flu Vaccine". cdc.gov. September 9, 2014. Retrieved 26 November 2014.

[5] http://www.who.int/influenza/vaccines/virus/recommen dations/201502_recommendation.pdf

[6] A. Combes *-, C.-E. Luyt, J.-L. Trouillet. Acute myocarditis. Réanimation 14 (2005) 248-254

[7] Giuseppe Rescigno*, Carlo Aratari, Marco L. S. Matteucci, Francesco Massi, Filippo Capestro, Alessandro D'Alfonso and Lucia Torracca, Management of transapical left venting during adult peripheral extracorporeal membrane oxygenation, Published: 28 March 2011

[8] Lin CH1, Chang JS, Li PC., The rescue of acute fulminant myocarditis by extracorporeal membrane oxygenation in pediatric patients.ActaPaediatr Taiwan. 2005 Jul-Aug;46(4):201-5.PMID: 16381333 [PubMed indexed for MEDLINE]

[9] A. COMBES, J.-L. TROUILLET, J. CHASTRE, C.-E. LUYT, Acute myocarditis, Urgences 2011, chapter 76.

[10] Kato S, Morimoto S, Hiramatsu S, Nomura M, Ito T, Hishida $H$. Use of percutaneous cardiopulmonary support of patients with fulminant myocarditis and cardiogenic shock for improving prognosis. Am J Cardiol 1999;83:623-625.

[11] Acker MA. Mechanical circulatory support for patients with acute-fulminant myocarditis. Ann ThoracSurg 2001;71:S73-S76.

[12] YasuhideAsaumi, Satoshi Yasuda, Isao Morii, Hiroyuki Kakuchi, Yoritaka Otsuka, Atsushi Kawamura, YoshikadoSasako, Takeshi Nakatani, Hiroshi Nonogi, Shunichi Miyazaki.

[13] Favourable clinical outcome in patients with cardiogenic shock due to fulminant myocarditis supported by percutaneous extracorporeal membrane oxygenation

DOI: http://dx.doi.org/10.1093/eurheartj/ehi411 2185-2192 First published online: 13 July 2005

[14]Yih-Sharng Chen* and Hsi-Yu Yu. Choice of mechanical support for fulminant myocarditis: ECMO vs. VAD?Corresponding author. yschen11@yahoo.com.tw (Y.-S. Chen) Received December 15, 2004. Accepted January 21, 2005.

[15] Chen YS, Yu HY, Huang SC, Chiu KM, Lin TY, Lai LP, Lin FY, Wang SS, Chu SH, Ko WJ. Experience and result extracorporeal membrane oxygenation in treating fulminant myocarditis with shock-what mechanical support should be considered first? J Heart Lung Transplant 2005;24(1):81-87.
[16] Grinda JM1, Chevalier P, D'Attellis N, Bricourt MO, Berrebi A, Guibourt P, Fabiani JN, Deloche A. Fulminant myocarditis in adults and children: biventricular assist device for recovery. Eur $\mathrm{J}$ Cardiothorac Surg. 2004 Dec;26(6):1169-73. 